\title{
MELHORAMENTO GENÉTICO DA CANA-DE-AÇÚCAR: AVALIAÇÃO DE CLONES PROVENIENTES DE HIBRIDAÇÕES EFETUADAS EM 1965 ( $\left.{ }^{1}\right)$
}

\author{
RAPHAEL ALVAREZ $\left({ }^{2}\right)$, ANTONIO LAZZARINI SEGALLA $\left({ }^{2}\right)$, \\ MARCOS GUIMARÃES DE ANDRADE LANDELL $(2,5)$, \\ MARIA BERNADETE SILVAROLLA $\left({ }^{3}\right)$ e GENTIL GODOY JUNIOR $\left({ }^{4}\right)$
}

\begin{abstract}
RESUMO
Objetivando estudar dezoito clones de cana-de-açúcar provenientes de hibridações efetuadas em Ubatuba, SP, em 1965, tendo como padrāo as variedades comerciais NA56-79 e CB41-76, efetuou-se um experimento em latossolo roxo na Usina Santa Lydia, em Ribeirão Preto, SP. No ensaio, plantado em março de 1973, utilizou-se o delineamento em blocos casualizados com quatro repetiçōes, sendo a análise estatística feita com a média das três colheitas (cana-planta, soca e ressoca). Avaliou-se a produção agrícola, teor de açúcar provável e produtividade de açúcar provável. O clone IAC65-55 apresentou produtividade de açúcar significativamente superior ao padrāo CB41-76, enquanto os clones IAC65-220, IAC65-257, IAC65-255, IAC65-155 e IAC65-113 não diferiram significativamente dele. Nenhum desses clones diferiu da variedade NA56-79 em produtividade de açúcar. Em função dessas características, associadas à resistência ao "carvão", os clones IAC65-55, IAC65-257, IAC65-113, IAC65-155 e IAC65-255 poderão constituir alternativas para o cultivo na regiāo de Ribeirão Preto.
\end{abstract}

Termos de indexaçã்o: cana-de-açúcar, melhoramento, hibridação; clones, avaliaçăo.

(1) Recebido para publicação em 7 de outubro de 1986.

(2) Seção de Cana-de-Açúcar, Instituto Agronómico (IAC), Caixa Postal 28, 13001 Campinas, SP.

(3) Seção de Genética, IAC.

(4) Estaçăo Experimental de Ubatuba, IAC.

(5) Com bolsa de suplementação do CNPq. 


\section{INTRODUÇÃo}

No Estado de São Paulo, o melhoramento genético da cana-de-açúcar, por via sexuada, vem sendo executado com regularidade pelo Instituto Agronômico desde 1947 (SEGALLA \& ALVAREZ, 1968). Como resultado dessas pesquisas, publicaram-se os seguintes trabalhos: ALVAREZ et al. $(1981,1983,1984$, 1986), SEGALLA \& ALVAREZ (1964) e SEGALLA et al. (1982).

Em 1965, a Seção de Cana-de-Açúcar realizou uma série de hibridações em Ubatuba, litoral norte paulista. $O$ presente trabalho refere-se à avaliação dos melhores clones provenientes dessa hibridação, feita no município de Ribeinão Preto, SP.

\section{MATERIAL E MÉTODOS}

No experimento, conduzido em latossolo roxo da Usina Santa Lydia, em Ribeinão Preto, utilizando-se delineamento em blocos ao acaso, com quatro repé tiçōes, estudaram-se os dezoito clones IAC abaixo, comparando-os aos padrōes NA56-79 e CB41-76.

\begin{tabular}{lllll}
\multicolumn{1}{c}{ Clones } & \multicolumn{1}{c}{$\begin{array}{c}\text { Progenitores } \\
\text { Cristalina } \times \text { Co285 }\end{array}$} & $\begin{array}{c}\text { Clones } \\
\text { IAC65-157 }\end{array}$ & \multicolumn{2}{c}{ POJ2878 $\times$ Co356 } \\
IAC65-9 & POJ2878 x Co285 & IAC65-168 & CB40-69 x & $?$ \\
IAC65-55 & POJ2878 x CB45-3 & IAC65-220 & Co419 x & $?$ \\
IAC65-83 & Co419 x IAC49-131 & IAC65-224 & Co419 x & $?$ \\
IAC65-87 & Co419 x IAC49-131 & IAC65-236 & Co419 x & $?$ \\
IAC65-99 & Co419 x IAC49-131 & IAC65-238 & Co419 x & $?$ \\
IAC65-113 & Co419 x Co356 & IAC65-241 & Co419 x & $?$ \\
IAC65-144 & Co419 x Co356 & IAC65-255 & Co419 x & $?$ \\
IAC65-145 & POJ2878 x ? & IAC65-257 & Co419 x & $?$
\end{tabular}

As parcelas foram constituídas de três linhas de $8,0 \mathrm{~m}$, espaçadas de $1,40 \mathrm{~m}$, com área útil de $33,60 \mathrm{~m}^{2}$. A adubação foi a mesma descrita em trabalhos anteriores (ALVAREZ et al., 1981, 1984).

Efetuou-se o plantio do experimento em março de 1973, com mudas de aproximadamente doze meses de idade, e três colheitas: a primeira, em meados do ano seguinte, com cerca de dezoito meses, e as subseqüentes, soca e ressoca, com intervalos de doze meses.

Nas colheitas, retiraram-se, de cada parcela, amostras de dez canas seguidas na linha, para análise do caldo. $O$ açúcar provável, em quilograma por tonelada de cana, foi calculado pela fórmula de Winter-Carp-Geerligs, modificada 
por ARCENEAUX (1935), com os fatores de correção 0,985 para brix e 0,970 para sacarose, e a eficiência das caldeiras modificada para $88 \%$, segundo AGUIRRE JUNIOR $\left({ }^{6}\right)$, para melhor adaptar os cálculos às condições das usinas paulistas.

Calculou-se a queda de produção da primeira para a terceira colheita pela expressão:

$$
Q=\frac{P_{1}-P_{3}}{P_{1}} \cdot 100
$$

onde:

$$
\begin{aligned}
P_{1}= & \text { produção da primeira colheita; } \\
P_{3}= & \text { produção da terceira colheita, conforme estabelecido por ALVA- } \\
& \text { REZ et al. (1984). }
\end{aligned}
$$

As análises estatisticas foram feitas para os dados de produtividade de cana (t/ha), teor de açúcar provável (kg açúcar/t cana) e produtividade de açúcar provável (t/ha), com a média das três colheitas.

\section{RESULTADOS E DISCUSSĀO}

Pelo quadro 1 - resultados da produtividade de cana e açúcar, e teor de açúcar provável - verifica-se que o clone IAC65-55 obteve produtividade de cana significativamente superior aos padrōes NA56-79 e CB41-76. Destacaram-se, também, os clones IAC65-238, IAC65-220, IAC65-236, IAC65-255, IAC65-257, IAC65-224 e IAC65-113, que não diferiram do IAC65-55 e dos padrões.

Quanto ao teor de açúcar provável, os clones IAC65-257, IAC65-144, IAC65-155, IAC65-145, IAC65-83, IAC65-220, IAC65-55 e IAC65-241 não diferiram da variedade NA56-79, que apresentou o maior teor e constitui o padrão superior para esse parâmetro. Os quatro primeiros clones foram estatisticamente superiores a CB41-76.

Em relação à produtividade de açúcar, o clone IAC65-55 foi significativamente superior à variedade CB41-76, não diferindo da NA56-79. Sobressairam-se, também, os clones IAC65-220, IAC65-257, IAC65-255, IAC65-155 e IAC65-113, que não diferiram estatisticamente do clone IAC65-55 e das testemunhas, apesar de seus valores absolutos serem superiores ao apresentado pela CB41-76.

Constam no quadro 2 os dados referentes à queda de produção da primeira (cana-planta) para a terceira (ressoca) colheita e da reação ao "carvão".

Pelos dados referentes ao. "carvão", observá-se que, dos dezoito clones em estudo, onze são resistentes e três são suscetiveis.

(6) AGUIRRE JUNIOR, J.M. Relatório da Seção de Cana-de-Açúcar de 1940. (Relatório interno) 
QUADRO 1. Produtividade de cana e de açúcar, teor de açúcar provável, e resumo da análise da variância dos dados do experimento conduzido com clones IAC provenientes de hibridaçōes realizadas em 1965, na Usina Santa Lydia, Ribeirāo Preto, SP

\begin{tabular}{lccc}
\hline Tratamentos & $\begin{array}{c}\text { Produtividade } \\
\text { de cana }\end{array}$ & $\begin{array}{c}\text { Teor } \\
\text { de açúcar }\end{array}$ & $\begin{array}{c}\text { Produtividade } \\
\text { de açúcar }\end{array}$ \\
\hline & t/ha & $\mathrm{kg} / \mathrm{t} \mathrm{cana}$ & $\mathrm{t} / \mathrm{ha}$ \\
IAC65-55 & $128,8 \mathrm{a}$ & $123,8 \mathrm{a}-\mathrm{c}$ & $15,8 \mathrm{a}$ \\
IAC65-220 & $121,4 \mathrm{ab}$ & $124,0 \mathrm{a}-\mathrm{c}$ & $15,1 \mathrm{ab}$ \\
IAC65-257 & $111,7 \mathrm{a}-\mathrm{c}$ & $126,8 \mathrm{ab}$ & $14,0 \mathrm{a}-\mathrm{c}$ \\
NA56-79 & $106,4 \mathrm{~b}-\mathrm{f}$ & $130,0 \mathrm{a}$ & $13,7 \mathrm{a}-\mathrm{d}$ \\
IAC65-255 & $112,3 \mathrm{a}-\mathrm{d}$ & $122,4 \mathrm{~b}-\mathrm{e}$ & $13,6 \mathrm{a}-\mathrm{d}$ \\
IAC65-155 & $106,1 \mathrm{~b}-\mathrm{f}$ & $126,2 \mathrm{ab}$ & $13,3 \mathrm{a}-\mathrm{e}$ \\
IAC65-113 & $110,5 \mathrm{a}-\mathrm{e}$ & $119,8 \mathrm{~b}-\mathrm{f}$ & $13,2 \mathrm{a}-\mathrm{e}$ \\
IAC65-236 & $115,3 \mathrm{a}-\mathrm{c}$ & $111,6 \mathrm{~g}$ & $12,9 \mathrm{~b}-\mathrm{e}$ \\
IAC65-238 & $121,6 \mathrm{ab}$ & $103,7 \mathrm{~h}$ & $12,6 \mathrm{~b}-\mathrm{f}$ \\
IAC65-241 & $101,3 \mathrm{~b}-\mathrm{g}$ & $123,3 \mathrm{a}-\mathrm{d}$ & $12,5 \mathrm{~b}-\mathrm{f}$ \\
CB41-76 & $105,7 \mathrm{~b}-\mathrm{f}$ & $117,7 \mathrm{c}-\mathrm{g}$ & $12,4 \mathrm{~b}-\mathrm{f}$ \\
IAC65-224 & $111,0 \mathrm{a}-\mathrm{e}$ & $111,3 \mathrm{~g}$ & $12,3 \mathrm{c}-\mathrm{f}$ \\
IAC65-4 & $101,6 \mathrm{~b}-\mathrm{g}$ & $116,5 \mathrm{~d}-\mathrm{g}$ & $11,8 \mathrm{c}-\mathrm{f}$ \\
IAC65-168 & $96,7 \mathrm{c}-\mathrm{g}$ & $116,0 \mathrm{e}-\mathrm{g}$ & $11,1 \mathrm{~d}-\mathrm{f}$ \\
IAC65-145 & $87,0 \mathrm{fg}$ & $125,6 \mathrm{ab}$ & $10,6 \mathrm{ef}$ \\
IAC65-144 & $82,6 \mathrm{~g}$ & $126,4 \mathrm{ab}$ & $10,5 \mathrm{ef}$ \\
IAC65-99 & $92,9 \mathrm{~d}-\mathrm{g}$ & $114,8 \mathrm{fg}$ & $10,5 \mathrm{ef}$ \\
IAC65-87 & $90,6 \mathrm{e}-\mathrm{g}$ & $115,4 \mathrm{e}-\mathrm{g}$ & $10,1 \mathrm{f}$ \\
IAC65-157 & $60,9 \mathrm{~h}$ & $120,4 \mathrm{~b}-\mathrm{f}$ & $7,3 \mathrm{~g}$ \\
IAC65-83 & $54,9 \mathrm{~h}$ & $124,7 \mathrm{a}-\mathrm{c}$ & $6,7 \mathrm{~g}$ \\
\hline F & $21,24^{* *}$ & $21,33^{\star *}$ & 2,7 \\
D.M.S. (Tukey 5\%) & 7,4 & 8,5 \\
C.V. \% & 21,5 & 2,3 & \\
\hline & $8, \mathrm{t}$ & & \\
\hline
\end{tabular}

Quanto à queda de produção da cana-planta para $2^{\mathrm{a}}$ soca, os clones IAC65-113, IAC65-236 e IAC65-220 mostraram-se superiores aos dois padrões. Essa caracteristica é muito importante quando se considera o manejo atual da cultura, que visa ao aumento do número de cortes (colheitas).

A análise conjunta das características agroindustriais permite as seguintes considerações: o clone IAC65-55 apresentou ótima produção agrícola, bom teor de açúcar e baixa queda de produção no decorrer das colheitas, nivelando-se, quanto a esse aspecto, à variedade NA56-79 e superando a CB41-76. Os clones IAC65-257 e IAC65-155 apresentaram, como características comuns, o alto teor 
de açúcar e a boa produção agrícola. O IAC65-255, com produção agrícola boa, revelou um teor de açúcar inferior ao da NA56-79. Comportamento semelhante apresentou o clone IAC65-113, que, no entanto, se destacou por ser o mais vigoroso nas socas. O clone IAC65-220, com ótima produção agrícola, bom teor de açúcar e bom vigor de socas, é suscetivel ao "carvão" da cana-de-açúcar.

Concluindo, em função das características agrotecnológicas e da resistência apresentada ao carvão, os clones IAC65-55, IAC65-257, IAC65-113, IAC65-155 e IAC65-255 poderão constituir alternativas para o cultivo na região de Ribeirão Preto.

QUADRO 2. Dados de queda da produção da $1^{\mathrm{a}}$ para a $3^{\mathrm{a}}$ colheita comparados pelo teste de Duncan a $1 \%$ de probabilidade, de reação ao carvão, e resumo da análise de variância

\begin{tabular}{|c|c|c|}
\hline & $\%$ & \\
\hline IAC65-113 $\ldots \ldots \ldots \ldots \ldots$ & $39,0 \mathrm{a}$ & $\mathrm{R}$ \\
\hline IAC65-236 $\ldots \ldots \ldots \ldots \ldots$ & $40,0 \mathrm{a}$ & $S$ \\
\hline IAC65-220 . . . . . . . . & $40,2 \mathrm{a}$ & $S$ \\
\hline IAC65-55 . . . . . . . & $43,0 a b$ & $\mathrm{R}$ \\
\hline IAC65-255 $\ldots \ldots \ldots \ldots \ldots$ & $45,9 a-c$ & $R$ \\
\hline IAC65-168 $\ldots \ldots \ldots \ldots \ldots$ & $46,0 a-c$ & $\mathrm{R}$ \\
\hline IAC65-238 $\ldots \ldots \ldots \ldots \ldots$ & $46,0 \mathrm{a}-\mathrm{c}$ & - \\
\hline IAC65-155 $\ldots \ldots \ldots \ldots$ & $46,9 a-c$ & R \\
\hline IAC65-224 $\ldots \ldots \ldots \ldots \ldots$ & $49,1 a-c$ & $\mathrm{R}$ \\
\hline IAC65-241 $\ldots \ldots \ldots \ldots \ldots$ & $51,4 \mathrm{bc}$ & $\mathrm{S}$ \\
\hline NA56-79 $\ldots \ldots \ldots \ldots \ldots$ & $51,5 \mathrm{bc}$ & I \\
\hline IAC65-144 $\ldots \ldots \ldots \ldots \ldots$ & $52,2 \mathrm{bc}$ & R \\
\hline IAC65-257 . . . . . . . . & $53,2 \mathrm{bc}$ & $\mathrm{R}$ \\
\hline CB41-76 $\ldots \ldots \ldots \ldots \ldots$ & $54,9 \mathrm{~cd}$ & $\mathrm{R}$ \\
\hline $\mid A C 65-4 \ldots \ldots \ldots \ldots \ldots$ & $56,0 c-e$ & - \\
\hline IAC65-99 $\ldots \ldots \ldots \ldots \ldots$ & $56,4 c-f$ & $\mathrm{R}$ \\
\hline IAC65-145 $\ldots \ldots \ldots \ldots$ & $64,4 d-f$ & - \\
\hline IAC65-157 ... . . . . . & 65,8 ef & - \\
\hline IAC65-87 ............ & $68,4 f$ & $\mathrm{R}$ \\
\hline IAC65-83 $\ldots \ldots \ldots \ldots \ldots$ & $68,6 \uparrow$ & R \\
\hline Valor de $F$ (tratamentos) . . . . . & $13,5^{\star \star}$ & \\
\hline CV $\% \ldots \ldots \ldots \ldots \ldots$ & 9,7 & \\
\hline
\end{tabular}

(1) Dados fornecidos pela Comissão de Controle do Carvão da Cana-de-Açúcar do Estado de São Paulo: $\mathrm{R}=$ resistente; $\mathrm{I}=$ intermediária; $\mathrm{S}=$ suscetlvel. 


\section{SUMMARY}

\section{SUGARCANE BREEDING: PERFORMANCE OF IAC SUGARCANE CLONES ORIGINATED FROM HYBRIDATION}

A group of 18 of the best sugarcane clones obtained from hybridation in 1965, at the Experimental Station of Ubatuba, Instituto Agronômico, were evaluated in one experiment carried out in Santa Lydia mill, Ribeirão Preto, State of Sāo Paulo, Brazil. In 1973, it was started a field trial using as controls the commercial varieties NA56-79, and CB41-76. The experiment design was a randomized complete block with four replications. The cane yield (t/ha), sugar content ( $\mathrm{kg} / \mathrm{t}$ cane), and sugar yield (t/ha) were expressed as the average of three harvests: cane plant (18 months), first ratoon (12 months after) and second ratoon (12 months after). According to these characteristics the clones IAC65-55, IAC65-257, IAC65-113, IAC65-155 and IAC65-255 were selected, and constitute alternatives of new varieties for the sugarcane cropping in the region of Ribeirão Preto.

Index terms: sugarcane, breeding, hybridation, evaluation of clones.

\section{REFERÊNCIAS BIBLIOGRÁFICAS}

ALVAREZ, R.; BASTOS, C.R.; LANDELL, M.G.A; BOVI, V.; POMMER, C.V.; PEREIRA, J.C.V.N.A.; SILVAROLLA, M.B.; GODOY JÜNIOR, G.; COSTA, A.A.; CAMARGO, A.P. \& RAMOS, M.T.B. Melhoramento da cana-de-açúcar. V. Experimentos regionais com clones obtidos em 1970. STAB, Piracicaba, 4(4):39-46, 1986.

- ; ; POMMER, C.V.; BRINHOLI, O.; DALBEN, A.E.; GODOY JÚNIOR, G.; BOVI, V. \& CIONE, J. Melhoramento da cana-de-açúcar. Ill. Experimentos regionais com clones obtidos em 1968. In: CONGRESSO NACIONAL DA SOCIEDADE DE TÉCNICOS AÇUCAREIROS DO BRASIL, 2., Rio de Janeiro, 1981. Anais. v.2, p.198-215.

; SEGALLA, A.L.; OLIVEIRA, H.; GODOY JÜNIOR, G.; POMMER, C.V.; BRINHOLI, O. \& DALBEN, A.E. Melhoramento da cana-de-açúcar. lla. Experimentos regionais com clones obtidos em 1967. Bragantia, Campinas, 42:27-36, 1983.

—; POMMER, C.V.; BASTOS, C.R.; BRINHOLI, O.; GODOY JÚNIOR, G. \& BOVI, V. Melhoramento da cana-de-açúcar. IV. Experimentos regionais com clones obtidos em 1969. Bragantia, Campinas, 43(1):155-165, 1984.

ARCENEAUX, G.L. A simplified method of theoretical sugar yield calculations. International Sugar Journat, 38:264-265, 1935.

SEGALLA, A.L. \& ALVAREZ, R. Melhoramento da cana-de-açúcar. I. Experiências com "seedlings" obtidos em 1947, 1948 e 1949. Bragantia, Campinas, 23:187-223, 1964.

\& - Realização do Instituto Agronômico. Contribuição ao desenvolvimento da cultura da cana-de-açúcar em Sāo Paulo. O Agronômico, Campinas, 20(5/6):1-35, 1968.

\& — OLIVEIRA, H.; IGUE, T. \& GODOY JÚNIOR, G. Melhoramento da cana-de-açúcar. II. Experimentos regionais com clones obtidos em 1964. Bragantia, Campinas, 41:109-117, 1982. 